\title{
Evaluation of Nasal and Temporal Anterior Chamber Angle with Four Different Techniques
}

\section{Fredrik P. Källmark, Mezghan Sakhi}

Unit of Optometry, Section of Ophthalmology and Vision, Department of Clinical Neurosience, Karolinska Instetutet, Stockholm, Sweden.

Email: fredrik.kallmark@eyelab.se

Received October $8^{\text {th }}, 2013$; revised November $5^{\text {th }}, 2013$; accepted December $2^{\text {nd }}, 2013$

Copyright (C) 2013 Fredrik P. Källmark, Mezghan Sakhi. This is an open access article distributed under the Creative Commons Attribution License, which permits unrestricted use, distribution, and reproduction in any medium, provided the original work is properly cited.

\begin{abstract}
Background: Anterior chamber angle (ACA) can be measured by many different techniques. In order for a technique to be a part of the routine eye examination, it has to be quick and easy in good agreement with gonioscopy both nasally and temporally. Aim: To investigate variation in ACA measurement between gonioscopy, van Herick technique, anterior segment optical coherence tomography (AS-OCT) and Sirius Scheimpflug-Camera both nasally and temporally. Method: The ACA of 50 eyes of 25 healthy subjects was measured with gonioscopy, van Herick technique, AS-OCT and Sirius Scheimpflug-Camera. The angle was measured both nasally and temporally. Results: No statistically significant difference could be found between gonioscopy, van Herick technique and AS-OCT either nasally or temporally. The Sirius Scheimpflug-Camera on the other hand showed statistically significant difference to gonioscopy ( $p<0.0001)$, van Herick technique $(p<0.0001)$ and AS-OCT $(p<0.0001)$ both nasally $(p=0.03, p=0.001, p<0.0001)$ and temporally $(\mathrm{p}=0.0002, \mathrm{p}=0.001, \mathrm{p}<0.0001)$. Conclusion: This study showed good agreement between three of the four techniques. ACA measurements obtained by the Sirius Scheimpflug-Camera should therefore not be considered interchangeable with those obtained by the remaining three methods.
\end{abstract}

Keywords: Anterior Chamber Angle (ACA); Gonioscopy; Van Herick Technique; Sirius Scheimpflug Camera; AS-OCT; Angle Closure Glaucoma

\section{Introduction}

Angle Closure Glaucoma (ACG), a sight threatening eye disease, is a major cause of blindness throughout the world [1].

ACG is caused by the abnormal positioning of the peripheral iris towards the trabecular meshwork which hinders the aqueous outflow resulting in an increased intraocular pressure (IOP), eventually leading to glaucomatous optic neuropathy and irreversible blindness [2-4].

Eyes with narrow angles and at risk of ACG can be prevented from the disease if they are identified prior to the disease's onset [5]. It is therefore very important to measure and evaluate the anterior chamber angle (ACA), Figure 1, of all the patients in a routine eye examination. One should also evaluate the ACA of elderly patients as the prevalence of ACG increases with age due to the gradual increase of the crystalline lens $[1,2,6]$. Evaluation of ACA should also be performed at patients who take medication that can cause pupil dilation [7]. For ACA evaluation in a routine eye examination, a quick and easy method is needed.

ACA can be measured by many different methods: e.g. gonioscopy, ultra sound biomicroscopy (UBM), van Herick technique, Scheimpflug photography, anterior segment optical coherence tomography (AS-OCT) and the Sirius Scheimpflug-Camera [1].

\subsection{Gonioscopy}

Gonioscopy, the current gold standard method, makes use of a slit lamp and a contact lens (gonioscopy lens) to view the ACA structures. When describing the angle with gonioscopy, several systems have been developed as Scheie, Shaffer, Becker and Spaeth $[8,10,11]$. Pressure on the gonioscopy lens and illumination used during the examination can alter the angle configuration. Furthermore, it is a time consuming subjective method that re- 


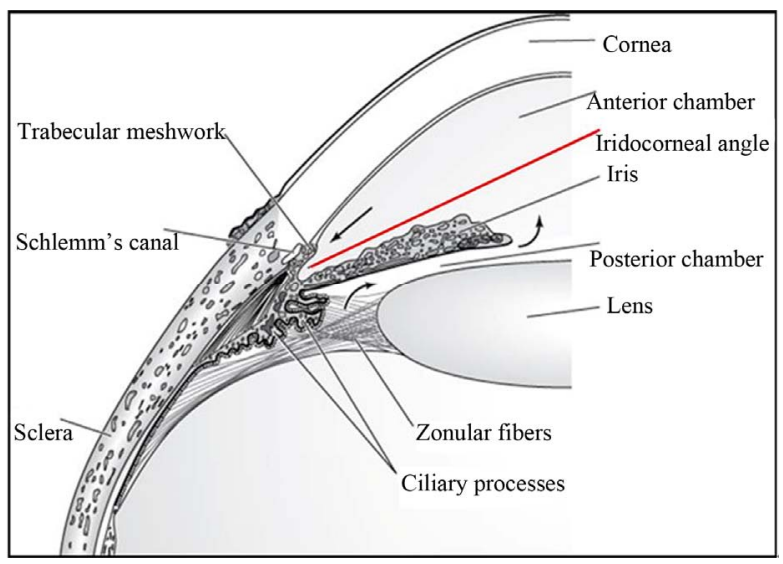

Figure 1. Diagram of the anatomical structures forming the iridocorneal angle.

quires considerable skill, knowledge and experience in order to achieve reliable measurements $[1,3,4,12]$. These limitations make gonioscopy less suitable as a quick evaluation method for ACA in a routine eye examination.

\subsection{Van Herick}

Van Herick technique, Sirius Scheimpflug-Camera and AS-OCT on the other hand can be part of a routine eye checkup as they all are quick and easy to perform and do not contact the cornea [1,7].

The van Herick technique uses a slit lamp to subjectively estimate the ACA whilst Sirius ScheimpflugCamera and AS-OCT provide objective measurement of the ACA.

\subsection{AS-OCT}

AS-OCT is a tomographic and biomicroscopic device of high resolution designed for imaging and measuring the anterior segment of the eye [3]. Measurement of the ACA by the AS-OCT takes less than a second. The ASOCT takes cross sectional images of the anterior segment that are analyzed by a semiautomatic software program that is connected to the device. The examiner marks the apex of the angle, the posterior surface of the cornea and the anterior surface of the iris and the software calculates the ACA in degrees $[3,13]$.

\subsection{Sirius Scheimpflug-Camera}

Sirius Scheimpflug-Camera is a combination of a 3D rotating Scheimpflug camera with a placido disc topographer that gives a detailed evaluation of the entire anterior eye segment including ACA. The scanning process takes less than one second and acquires about 25 Scheimpflug images, which are analyzed by the computer software program that is connected to the device and the ACA is presented in degrees [14].

\section{Previous Comparisons}

Good agreement between gonioscopy and the van Herick technique is reported in a study by Kashiwagi et al. and in another study by Foster et al. $[15,16]$. Conversely, in a study by Thomas et al. the van Herick technique is said to be in disagreement with gonioscopy because it measures the angle wider than gonioscopy [17]. AS-OCT is reported to detect more closed ACAs than gonioscopy in a study by Sakata et al. [18]. Patients with narrow angle and those at risk of ACG can be missed if the angle is measured wider than its real size. If the angle on the other hand is measured narrower than its actual size, additional evaluation by gonioscopy is required, which will incur unnecessarily costs to the health care system. A quick and accurate ACA evaluation method is therefore necessary.

Since the Sirius Scheimpflug-Camera is a recently developed device, limited number of studies has been performed on it. High repeatability for the anterior segment measurement by the Sirius Scheimpflug-Camera is reported in a study by Savini et al. [19]. However, the study does not state anything about the repeatability of the ACA measurement by the device. In addition, to the best of our knowledge, no study comparing the Sirius Scheimpflug-Camera to gonioscopy, the van Herick technique and the AS-OCT has yet been performed.

Most of the previous studies compare the temporal ACA findings of van Herick technique to that of gonioscopy $[5,12,13]$. In a study by Pettersson and Källmark and in another study by Friedman et al. significant difference between temporal and nasal ACA is reported $[1,20]$. For an accurate comparison of ACA measuring techniques, the angle should therefore be measured both nasally and temporally as the techniques can be in agreement nasally but in disagreement temporally or vice versa.

\section{Aims}

The aim of this study was to investigate variation in ACA measurement between gonioscopy, van Herick technique, AS-OCT and Sirius Scheimpflug-Camera both nasally and temporally

\section{Methods and Material}

\subsection{Patients and Clinical Investigation}

ACA of both eyes of 25 randomly selected subjects (mean age 24.28 \pm 3.77 ) were measured with all the four methods (i.e. gonioscopy, van Herick technique, Sirius Scheimpflug-Camera and AS-OCT). The study was performed at S:t Erik Eye hospital and in accordance with the declaration of Helsinki. Informed consent was obtained from all the participants after they were well in- 
formed of the study's nature. The ACA measurement obtained with van Herick technique and gonioscopy was carried out by an experienced optometrist masked to the test results obtained by the Sirius Scheimpflug-Camera and the AS-OCT. The Sirius Scheimpflug-Camera and the AS-OCT measurements were performed by another optometrist who was unaware of the gonioscopy and the van Herick results. On the day the angle measures were carried out, subjects had been free from contact lens wear.

\subsection{Van Herick Technique}

ACA was measured both nasally and temporally with the slit lamp using van Herick technique. The ACA was estimated by comparing the limbal anterior chamber depth (LACD) (which was observed as an empty space between the corneal endothelium and the anterior iris) to the corneal thickness. ACA was graded according to the van Herick's grading system which is illustrated in Table $\mathbf{1}[1,3,7,21]$.

\subsection{Gonioscopy}

Gonioscopy was performed using a Goldman 3-mirror lens. The cornea was anesthetized using one drop of $0.4 \%$ oxybuprocaine hydrochloride. Two drops of $2 \%$ methylcellulose were used as a coupling medium for the gonioscopy lens. Care was taken to avoid accidental indentation of the angle by direct illumination of the pupil during the examination. Slight tilting of the lens was allowed to gain view over the convexity of the iris. The ACA was graded using Shaffer grading system which is illustrated in Table $2[1,4,6,13,22]$.

\subsection{AC-OCT}

The AS-OCT used in this study was Visante OCT manufactured by Carl Zeiss Meditec. The nasal and temporal ACA can be measured simultaneously by the AS-OCT. Images of the ACA were acquired with the subject seated and fixating an internal fixation target. The subject was instructed to blink and open his/her eye widely before

Table 1. ACA grading system as described by van Herik.

\begin{tabular}{ccc}
\hline $\begin{array}{c}\text { Width of the empty space } \\
\text { (LACD }) \text { as compared to the } \\
\text { corneal thickness }\end{array}$ & $\begin{array}{c}\text { van Herick } \\
\text { Grade }\end{array}$ & Angle status \\
\hline $\begin{array}{c}\text { No black space observed } \\
<1 / 4 \text { Corneal thickness }\end{array}$ & 0 & Closed \\
$1 / 4$ of corneal thickness & 2 & Extremely narrow \\
$>1 / 4$ to $1 / 2$ of corneal thickness & 3 & Narrow \\
$\geq 1$ of corneal thickness & 4 & Wide open \\
\hline
\end{tabular}

${ }^{*}$ LACD $=$ Limbal Anterior Chamber Depth.
Table 2. ACA grading system as described by Shaffer.

\begin{tabular}{cccc}
\hline $\begin{array}{c}\text { ACA* } \\
\text { in degrees }\end{array}$ & ACA grade & Angle status & Visible structures \\
\hline 0 & 0 & Closed & No structures visible \\
$\leq 10$ & 1 & Extremely narrow & Schwalbe's line \\
$11-19$ & 2 & Narrow & Trabecular meshwork \\
$20-34$ & 3 & Open & Scleral spur \\
$35-45$ & 4 & Wide open & Ciliary body \\
\hline${ }^{*}$ ACA $=$ Anterior Chamber Angle. & &
\end{tabular}

each measurement. Acquired images were then analysed using the semi-automated software programme provided by the device.

\subsection{Sirius Scheimpflug-Camera}

The Sirius Scheimpflug-Camera gives values of both the nasal and the temporal ACA in a single measurement. The central fixation target of the device was used to align the subject's eye along the visual axis and to bring the device into focus. The subject was instructed to blink and open his/her eye widely before each measurement. Three consecutive measurements were carried out per eye and a mean value of the ACA was calculated which was taken as the single measured value that was compared to the values obtained by the other methods. Phoenix SoftwareSuite was used by the device in this study to analyse the obtained Scheimpflug images.

\subsection{Angle Conversion}

As stated before, both the AS-OCT and the Sirius Scheimpflug-Camera measure the ACA in degrees and can therefore not be directly compared to the van Herick technique and gonioscopy. Hence the ACA values obtained with these two devices were converted to the van Herick's grading system using Shaffer's anterior chamber angle grading system (illustrated in Table 2). Nevertheless, both AS-OCT and the Sirius ScheimpflugCamera measure the ACA in degrees so comparisons between them were performed in degrees.

\subsection{Statistical Analysis}

The Graph pad Instat for windows (Version 3.10) was used for statistical analysis. The Friedman test with posttest was used for comparison between two different methods. The Wilcoxon matched pair test was used for either nasal or temporal data comparison between two methods. A p value less than $0.05(\mathrm{p}<0.05)$ was considered statistically significant and indicated a difference between the measurements. 


\section{Results}

The descriptive statistics for nasal and temporal measurements is shown in Table 3.

The mean ACA measured by gonioscopy and the van Herick technique differed by 0.12 nasally and by 0.04 temporally. No statistically significant difference could be found between these two methods $(p=0.45)$ either nasally $(\mathrm{p}=0.20)$ or temporally $(\mathrm{p}=0.68)$. Figure 1 compares measurements obtained by gonioscopy to that obtained by van Herick technique.

On comparison of the mean ACA values obtained by gonioscopy to that obtained by the AS-OCT a difference of 0.14 was observed nasally and a difference of 0.08 was observed temporally. But no statistically significant difference could be found between the two methods $(\mathrm{p}=$ $0.09)$ neither nasally $(p=0.15)$ nor temporally $(p=0.36)$. A comparison of AS-OCT findings to that of gonioscopy is shown in Figure 2.

When the mean ACA value obtained by gonioscopy is compared to that obtained by the Sirius ScheimpflugCamera a difference of 0.18 was seen between nasal values and a difference of 0.30 was seen between temporal

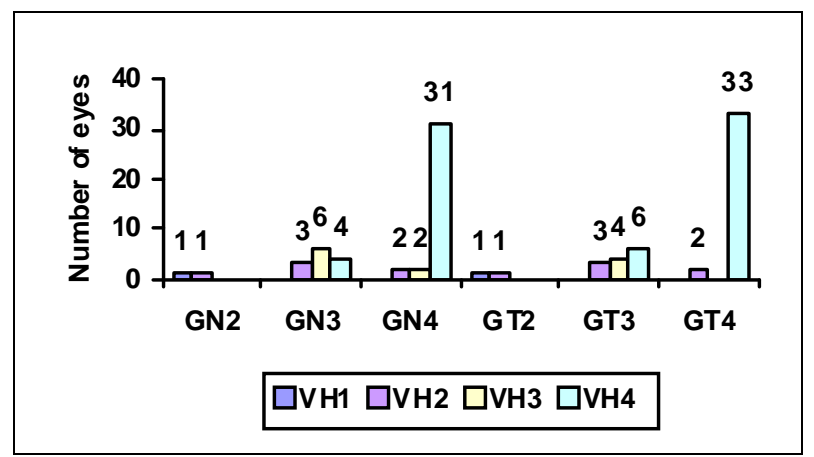

Figure 2. Compares the measured ACA grading's obtained by gonioscopy to that obtained by van Herick technique. Numbers over the bars; show the number of eyes, GN2 (gonioscopy nasal grade 2), GN3 (gonioscopy nasal grade 3), GN4 (gonioscopy nasal grade 4), GT2 (gonioscopy temporal grade 2), GT3 (gonioscopy temporal grade 3), GT4 (gonioscopy temporal grade 4), VH1, 2, 3 and 4 (van Herick grade 1, 2, 3 and 4).

Table 3. Descriptive statistics for nasal and temporal measurements.

\begin{tabular}{ccccc}
\hline & Gonio-scopy & van Herick & AS-OCT $^{*}$ & Sirius $^{* *}$ \\
\hline Mean & $3.66 / 3.66$ & $3.54 / 3.62$ & $3.52 / 3.58$ & $3.84 / 3.96$ \\
SD $^{* * *}$ & $0.56 / 0.56$ & $0.79 / 0.78$ & $0.50 / 0.50$ & $0.37 / 0.20$ \\
$\begin{array}{c}95 \% \\
\text { confidence } \\
\text { interval }\end{array}$ & $0.159 / 0.159$ & $0.224 / 0.222$ & $0.144 / 0.142$ & $0.105 / 0.056$ \\
Range & $2-4$ & $1-4$ & $3-4$ & $3-4$ \\
\hline
\end{tabular}

*Anterior segment optical coherence tomography. ${ }^{* *}$ Sirius scheimpflugCamera. ${ }^{* * *}$ Standard deviation. values. A statistically significant difference was found between these two methods $(p<0.0001)$ both nasally ( $p$ $=0.03)$ and temporally $(\mathrm{p}=0.0002)$. Values obtained by Sirius Scheimpflug-Camera are compared to that obtained by gonioscopy in Figure 3.

A difference of 0.02 between the mean nasal measurements and a difference of 0.04 between the mean temporal measurements of the van Herick technique and the AS-OCT was found. No statistically significant difference was found to exist between the two methods $(\mathrm{p}=$ $0.39)$ either nasally $(p=0.86)$ or temporally $(p=0.71)$. Figure 4 shows a comparison of these two methods.

The mean ACA value obtained nasally by the van Herick technique and the Sirius Scheimpflug-Camera differed by 0.30 whilst that obtained temporally differed by 0.34 . The two methods were found to be significantly different from each other $(\mathrm{p}<0.0001)$ both nasally $(\mathrm{p}=$ $0.001)$ and temporally $(\mathrm{p}=0.001)$. A comparison between these two methods.

The mean ACA value obtained nasally by the van

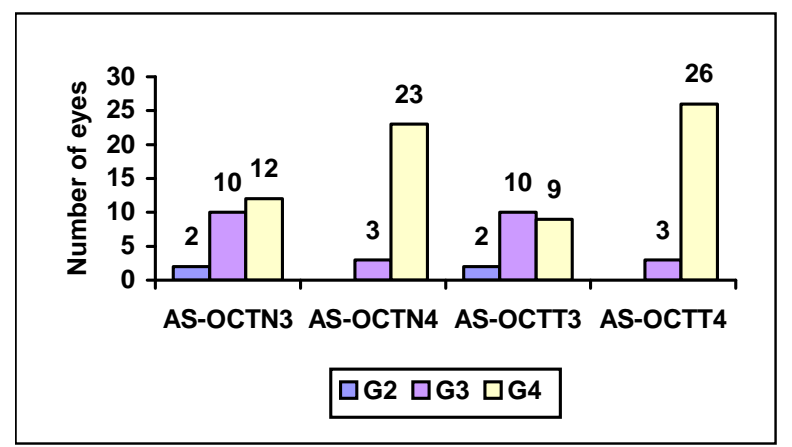

Figure 3. Compares the measured ACA grading's obtained by AS-OCT to that obtained by gonioscopy. Numbers over the bars, show the number of eyes AS-OCTN3 (AS-OCT nasal grade 3) AS-OCTN4 (AS-OCT nasal grade 4) ASOCTT3 (AS-OCT temporal grade 3) AS-OCTT4 (AS-OCT temporal grade 4) G2, 3 and 4 (gonioscopy grade 2, 3 and 4).

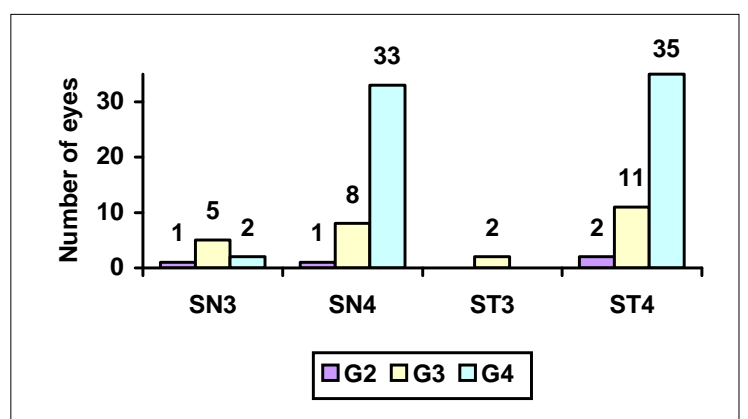

Figure 4. Compares the measured ACA grading obtained by Sirius Scheimpflug-Camera to that obtained-by gonioscopy. Numbers over the bars, show the number of eyesSN3 (Sirius Scheimpflug-Camera nasal grade 3) SN4 (Sirius Scheimpflug-Camera nasal grade 4) ST3 (Sirius Scheimpflug-Camera temporal grade 3) ST4 (Sirius Scheimpflug-Camera temporal grade 4. 
Herick technique and the Sirius Scheimpflug-Camera differed by 0.30 whilst that obtained temporally differed by 0.34 . The two methods were found to be significantly different from each other $(p<0.0001)$ both nasally $(p=$ $0.001)$ and temporally $(p=0.001)$. A comparison between these two methods is illustrated in Figure 5.

Table 4 shows the descriptive statistic in degrees for nasal and temporal measurements obtained by the ASOCT and the Sirius Scheimpflug-Camera. When comparing the mean ACA value obtained by the AS-OCT to that obtained by the Sirius Scheimpflug-Camera a difference of $7.84^{\circ}$ was observed nasally and a difference of $10.88^{0}$ was observed temporally. Thus a statistically significant difference was found to exist between these two methods ( $p<0.0001)$ both nasally $(\mathrm{p}<0.0001)$ and temporally $(\mathrm{p}<0.0001)$. Values obtained by these two methods are compared to each other in Figure 6.

\section{Discussion}

To decrease the risk for irreversible blindness due to ACG, a quick and easy ACA evaluation method is needed that should be a part of the routine eye examination.

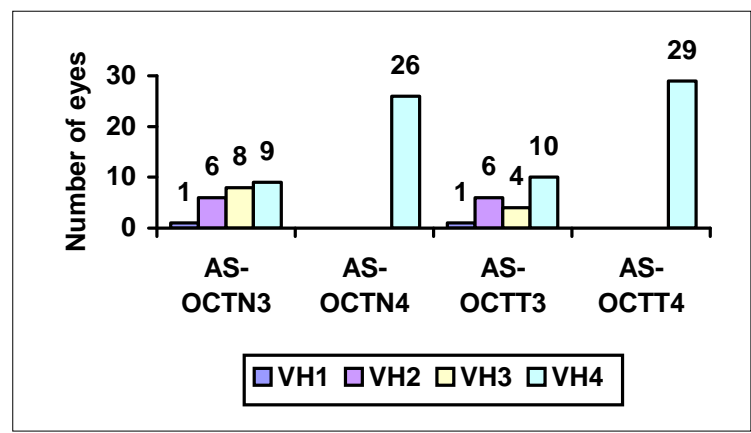

Figure 5. Compares the measured ACA grading's obtained by the AS-OCT to that obtained by van Herick technique. Numbers on the bars, show the number of eyes. AS-OCTN3 (AS-OCT nasal grade 3) AS-OCTN 4 (AS-OCT nasal grade 4) AS-OCTT3 (AS-OCT temporal grade 3) AS-OCTT4 (AS-OCT temporal grade 4) VH1, 2, 3 and 4 (van Herick grade 1, 2, 3 and 4).

Table 4. ACA measurement in degrees obtained by ASOCT and the Sirius Scheimpflug-camera.

\begin{tabular}{ccccc}
\hline & $\begin{array}{c}\text { AS-OCT }^{*} \\
\text { nasal } \\
\text { (in degrees) }\end{array}$ & $\begin{array}{c}\text { Sirius }^{* *} \\
\text { nasal } \\
\text { (in degrees) }\end{array}$ & $\begin{array}{c}\text { AS-OCT } \\
\text { temporal } \\
\text { (in degrees) }\end{array}$ & $\begin{array}{c}\text { Sirius }^{* *} \text { temporal } \\
\text { (in degrees) }\end{array}$ \\
\hline Mean & 35.86 & 43.70 & 37.66 & 48.53 \\
$\mathrm{SD}^{* * *}$ & 7.059 & 5.819 & 5.793 & 7.042 \\
$\begin{array}{c}95 \% \\
\text { confidence } \\
\text { interval }\end{array}$ & 1.66 & 2.01 & 2.00 & 1.64 \\
Range & $26-49.2$ & $30-61.67$ & $27-53.3$ & $34-65$ \\
\hline
\end{tabular}

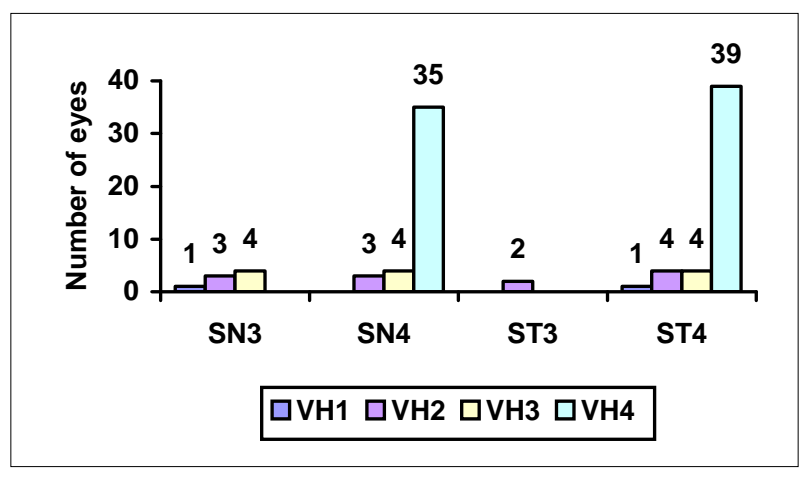

Figure 6. Compares the measured ACA grading's obtained by Sirius Scheimpflug-Camera to that obtained by van Herick technique. Numbers on the bars, show the number of eyes. SN3 (Sirius Scheimpflug-Camera nasal grade 3) SN4 (Sirius Scheimpflug-Camera nasal grade 4) ST3 (Sirius Scheimpflug-Camera temporal grade 3) ST4 (Sirius Scheimpflug-Camera temporal grade 4) VH1, 2, 3 and 4 (van Herick grade 1, 2, 3 and 4).

The van Herick technique, AS-OCT and Sirius Scheimpflug-Camera can be used for this purpose as they all are quick and easy to perform. But we need to know which one of these is the most accurate one to be used. To answer this question they have to be compared to gonioscopy which is the current reference standard for ACA measurement. This study was therefore carried out to investigate variation between these four different methods in measuring the ACA.

Our study showed good agreement between gonioscopy and the van Herick technique since no statistically significant difference could be found between the two methods $(p=0.45)$ either nasally $(p=0.20)$ or temporally $(p=0.68)$. Best agreement was shown for angles that had grade 4 since the majority of the eyes graded as 4 by gonioscopy were also graded as 4 by van Herick technique both nasally and temporally (Figure 1). This finding is in good agreement with the results of the study by Foster et al. where they also show good agreement between gonioscopy and van Herick technique especially for eyes having grade 4 [15].

Good agreement between gonioscopy and van Herick technique is also reported in a study by Park et al. and in another study by Kashiwagi et al. and in one other study by Foster et al. $[3,15,16]$. However, the result of our study disagrees with that of the study by Thomas et al. where poor agreement between gonioscopy and van Herick technique is reported. Difference in the study population between the two studies may explain this disagreement. Most of the subjects enrolled in their study had narrow angles whilst most of the subjects enrolled in our study had normal angles.

Both gonioscopy and the van Herick technique make use of the slit lamp to measure the ACA and both are subjective methods. These similarities can be the reason 
for good agreement between the two methods.

Our findings suggest poor agreement between gonioscopy and the Sirius Scheimpflug-Camera $(\mathrm{p}<0.0001)$ both nasally $(p=0.03)$ and temporally $(p=0.0002)$. No studies comparing Sirius Scheimpflug-Camera to gonioscopy has been performed before. Though, in a study by Friedman et al., the ACA measured by Scheimpflug camera is compared to the measurements obtained by gonioscopy and poor agreement between the two is reported [23].

Statistically significant difference between the results obtained by the Sirius Scheimpflug-Camera and the van Herick technique exists according to this study, indicating poor agreement between the two methods $(\mathrm{p}<$ $0.0001)$ both nasally $(\mathrm{p}=0.001)$ and temporally $(\mathrm{p}=$ $0.001)$.

Sirius Scheimpflug-Camera is in disagreement with gonioscopy and the van Herick technique may be because of its different nature compared to the other two methods. Both gonioscopy and the van Herick technique are subjective methods whilst the Sirius ScheimpflugCamera gives an objective measurement of the ACA. As stated before, both gonioscopy and the van Herick technique make use of the slit lamp whilst the Sirius Scheimpflug-Camera uses the 3D rotating Scheimpflug camera and bases its measurement results on the pictures obtained by that camera. In addition, the illumination used by the topographic rings and the fixation target of the Sirius Scheimpflug-Camera is a bit bright. This brightness can cause pupil constriction, which increases the angle width and there by a wider angle measure is given.

This study also showed good agreement between the results obtained by gonioscopy and the AS-OCT ( $\mathrm{p}=$ $0.09)$ for both nasal $(\mathrm{p}=0.15)$ and temporal data $(\mathrm{p}=$ 0.36). In a study by Sakata et al. poor agreement between gonioscopy and AS-OCT is reported for superior and inferior angles, but the agreement for nasal and temporal angle is reported to be good [18]. They also stated that AS-OCT tends to detect more closed ACAs than gonioscopy which can partly be seen in our study too. When comparing the mean ACA values obtained by gonioscopy to that obtained by AS-OCT, it can be seen that AS-OCT measures the angle 0.14 nasally and 0.08 temporally narrower than gonioscopy.

Our study showed good agreement between the ASOCT and the van Herick technique $(\mathrm{p}=0.39)$ both nasally $(\mathrm{p}=0.86)$ and temporally $(\mathrm{p}=0.71)$. However, AS-OCT is reported to be in disagreement with gonioscopy and van Herick technique in a study by Park et al. [3]. Eyes with narrow angles were used in their study whilst eyes with normal angles were used in our study. This difference in the selection of eyes in the two studies might be the reason for the disagreement between them.
AS-OCT also measures the ACA objectively but unlike Sirius Scheimpflug-Camera, it is in good agreement with both gonioscopy and the van Herick technique. This can partly be explained by the fact that both gonioscopy and the AS-OCT make a direct view of the ACA configuration to measure it. Also the AS-OCT makes use of an internal fixation target of low illumination to compensate for the subject's refractive error as well as prevent pupil constriction. Compensation of the refractive error is essential for prevention of the pupil constriction and lens disposition which normally occurs as a result of accommodation [24].

According to this study the results obtained by the AS-OCT differ significantly from those obtained by the Sirius Scheimpflug-Camera $(p<0.0001)$ both nasally ( $p$ $<0.0001)$ and temporally $(\mathrm{p}<0.0001)$ showing disagreement between the two devices.

The Sirius Scheimpflug-Camera makes use of visible light to measure the angle and can thus alter the angle configuration whilst the AS-OCT makes use of infrared light for its angle measurement. Besides, the Sirius Scheimpflug-Camera does not make any adjustment for the subject's refractive error and makes use of quite bright illumination in its fixation target and in its topographic rings. The AS-OCT on the other hand takes all of these factors in to account, which may thus explain the existence of poor agreement between the two devices.

To state which one of the three methods (i.e. van Herick technique, AS-OCT and Sirius ScheimpflugCamera) is the most accurate one to be used, is difficult because all of these methods have their own strengths and weaknesses. For instance, although the van Herick technique is a quick method that makes use of a slit lamp (which is available to all the optometrists and does not cost very much), it requires experience and good measuring skill to get reliable measurements [1]. Furthermore, it is a subjective method that gives an estimation of the angle as well as affected by the corneal thickness [4].

In contrast, measuring the ACA with the AS-OCT and Sirius Scheimpflug-Camera is not only quick but also easy to perform. In addition, they do not require much of experience and measuring skill and are objective methods. Nevertheless, the weakness of these devices is their high cost.

This study did have limitations. The illumination of the room which plays a vital role on the pupil constriction and dilation was not controlled in this study. At times the ACA was measured in a totally dark room and at times it was measured in a dimly illuminated room. This should, however, not affect the results enormously since light falling directly on the pupil affects its size more than the room illumination. And the light directly falling on the pupil was from the illumination used by the slit lamp and the fixation target used by the Sirius 
Scheimpflug-Camera and the AS-OCT. The measurements by gonioscopy and the van Herick technique were carried out by the same examiner which might have produced systemic bias. The number of subjects used in this study was not very big, so in order to get more reliable results the study should be performed on a larger group of people. In spite of these limitations, this study to the best of our knowledge is the first one to compare the Sirius Scheimpflug-Camera with gonioscopy, the van Herick technique and the AS-OCT.

\section{Conclusion}

In summary, this study showed good agreement between gonioscopy, the van Herick technique and the AS-OCT. The agreement between the Sirius Scheimpflug-Camera and the other three methods (i.e. gonioscopy, van Herick technique and AS-OCT) on the other hand was poor. It should therefore be noted that the ACA measurements obtained by the Sirius Scheimpflug-Camera, which cannot be considered interchangeable with those obtained by gonioscopy, the van Herick technique and the AS-OCT.

\section{REFERENCES}

[1] D. S. Friedman and M. He, "Anterior Chamber Angle Assessment Techniques," Survey of Ophthalmology, Vol. 53, No. 3, 2008, pp. 250-273. http://dx.doi.org/10.1016/j.survophthal.2007.10.012

[2] L. A. Remington, "Clinical Anatomy of the Visual System," 2nd Edition, Butterworth-Heinemann, Oxford, 2004.

[3] S. B. Park, K. R. Sung, S. Y. Kang, J. W. Jo, K. S. Lee and M. S. Kook, "Assessment of Narrow Angles by Gonioscopy, Van Herick Method and Anterior Segment Optical Coherence Tomography," Japanese Journal of Ophthalmology, Vol. 55, No. 4, 2011, pp. 343-350. http://dx.doi.org/10.1007/s10384-011-0036-0

[4] S. H. Kim J. H. Kang, K. H. Park and C. Hong, "Hong's Grading for Evaluating Anterior Chamber Angle Width," Japanese Journal of Ophthalmology, Vol. 56, No. 6, 2012, pp. 551-558.

http://dx.doi.org/10.1007/s10384-012-0180-1

[5] W. Nolan, P. Foster, J. Devereux and D. Uranchimeg, "YAG Laser Iridotomy Treatment for Primary Angle Closure in East Asian Eyes," The British Journal of Ophthalmology, Vol. 84, No. 11, 2000, pp. 1255-1259. http://dx.doi.org/10.1136/bjo.84.11.1255

[6] T. Grosvenor, "Primary Care Optometry," 5th Edition, Butterworth-Heinemann, Oxford, 2006.

[7] D. B. Elliot, "Clinical Procedures in Primary Eye Care," 3rd Edition, Butterworth-Heinemann, Oxford, 2007.

[8] H. G. Scheie, "Width and Pigmentation of the Angle of the Anterior Chamber Depth Assessment in Glaucoma Screening," Eye, Vol. 19, 2005, pp. 990-994.

[9] R. N. Shaffer, "Primary Glaucomas. Gonioscopy, Ophthalmoscopy and Perimetry," Transactions of the American Academy of Ophthalmology, Vol. 64, 1960, pp. 112-
127.

[10] S. C. Becker, "Clinical Gonioscopy: A Text and Stereoscopic Atlas," St. Louis. Mo, CV Mosby, 1972.

[11] G. L. Spaeth, "The Normal Development of the Human Anterior Chamber Angle: A New System of Descriptive Grading," Transactions of the Ophthalmological Societies of the United Kingdom, Vol. 91, 1971, pp. 709-739.

[12] K. Kashiwagi, T. Tokunaga, A. Iwase and T. Yamamoto, "Usefulness of Peripheral Anterior Chamber Depth Assessment in Glaucoma Screening," Eye, Vol. 19, 2005, p. 990. http://dx.doi.org/10.1038/sj.eye.6701700

[13] R. S. Chong, L. M. Sakata, S. W. Ho, M. He, M. Baskaran, T. Y. Wong, S. A. Perera and T. Aung, "Relationship between Intraocular Pressure and Angle Configuration: An Anterior Segment OCT Study," Investigative Ophthalmology \& Visual Science, Vol. 54, No. 3, 2013, pp. 1650-1655.

http://dx.doi.org/10.1167/iovs.12-9986

[14] G. Savini, M. Carbonelli, A. Sbreglia, P. Barboni, G. Deluigi and K. J. Hoffer, "Comparison of Anterior Segment Measurements by 3 Scheimpflug Tomographers and 1 Placido Corneal Topographer," Journal of Cataract \& Refractive Surgery, Vol. 37, No. 9, 2011, pp. 1679-1685. http://dx.doi.org/10.1016/j.jcrs.2011.03.055

[15] P. Foster, J. Devereux, P. H. Alsbirk and P. S. Lee, "Detection of Gonioscopically Occludable Angles and Primary Angle Closure Glaucoma by Estimation of Limbal Chamber Depth in Asians: Modified Grading Scheme," The British Journal of Ophthalmology, Vol. 84, No. 2, 2000, pp. 186-192. http://dx.doi.org/10.1136/bjo.84.2.186

[16] K. Kashiwagi, T. Tokunaga, A. Iwase, T. Yamamoto and S. Tsukahara, "Agreement between Peripheral Anterior Chamber Depth Evaluation Using the Van Herick Technique and Angle Width Evaluation Using the Shaffer System in Japanese," Japanese Journal of Ophthalmology, Vol. 49, No. 2, 2005, pp. 134-136. http://dx.doi.org/10.1007/s10384-004-0169-5

[17] R. Thomas, T. George, A. Braganza and J. Muliyil, "The Flashlight Test and Van Herick's Test Are Poor Predictors for Occludable Angles," Australian and New Zealand Journal of Ophthalmology, Vol. 24, No. 3, 1996, pp. 251256.

http://dx.doi.org/10.1111/j.1442-9071.1996.tb01588.x

[18] L. M. Sakata, R. Lavanya D. S. Friedman, H. Aung Gao, R. S. Kumar, P. J. Foster and T. Aung, "Comparison of Gonioscopy and Anterior Segment Ocular Coherence Tomography Detecting Angle Closure in Different Quadrants of the Anterior Chamber Angle," The American Academy of Ophthalmology, Vol. 115, No. 5, 2008, pp. 769-774. http://dx.doi.org/10.1016/j.ophtha.2007.06.030

[19] G. Savini, P. Barboni, M. Carbonelli and K. J. Hoffer, "Repeatability of Automatic Measurements by a New Scheimpflug Camera Combined with Placido Topography," Journal of Cataract \& Refractive Surgery, Vol. 37, No. 10, 2011, pp. 1809-1816. http://dx.doi.org/10.1016/j.jcrs.2011.04.033

[20] L. Pettersson and F. Källmark, "Difference in the Anterior Chamber Angle of the Four Meridians," Journal of Applied Medical Sciences, Vol. 1, No. 1, 2012, pp. 1-13. 
[21] F. Eperjesi and C. Holden, "Comparison of Techniques for Measuring Anterior Chamber Depth: Orbscan Imaging, Smith's Technique, and van Herick's Method," Graefe's Archive for Clinical and Experimental Ophthalmology, Vol. 249, No. 3, 2011, pp. 449-454. http://dx.doi.org/10.1007/s00417-010-1500-0

[22] K. Kashiwagi, T. Tsumura and S. Tsukahara, "Comparison between Newly Developed Scanning Peripheral Anterior Chamber Depth Analyzer and Conventional Methods of Evaluating Anterior Chamber Configuration," Journal of Glaucoma, Vol. 15, No. 5, 2006, pp. 380-387. http://dx.doi.org/10.1097/01.ijg.0000212254.17950.0b
[23] D. S. Friedman, G. Gazzard, C. B. Min and A. T. Broman, "Age and Sex Variation in Angle Findings among Normal Chinese Subjects: A Comparison of UBM, Scheimpflug, and Gonioscopic Assessment of the Anterior Chamber Angle," Journal of Glaucoma, Vol. 17, No. 1, 2008, pp. $5-10$. http://dx.doi.org/10.1097/IJG.0b013e31806ab327

[24] C. K. Leung and R. N. Weinreb, "Anterior Chamber Angle Imaging with Optical Coherence Tomography," Eye, Vol. 25, 2011, p. 261. http://dx.doi.org/10.1038/eye.2010.201 